\title{
The influence of the extraction method on the content of silymarin in Silybi mariani fructus
}

\author{
SofiJa ĐorĐeVIć ${ }^{1, *}$, TeOdora Janković ${ }^{1}$, AND Milica MihaIlović ${ }^{1}$ \\ ${ }^{1}$ Institute for Medicinal Plant Research "Dr. Josif Pančić”, Tadeuša Košćuška 1, 11000 Belgrade, Serbia \\ *Corresponding author: sdjordjevic@mocbilja.rs
}

Received: November 22, 2018

Accepted: December 10, 2018

Published on-line: December 20, 2018

Published: December 25, 2018

\begin{abstract}
This study was aimed to compare the efficiency of silymarin extraction from Silybi mariani fructus using different extraction methods. Maceration, percolation, extraction in a water bath, and ultrasonicassisted extraction have been performed with $60 \%$ ethanol. Extracts were analyzed using UV/Vis and HPLC techniques. Our results showed that the highest concentration of silymarin was detected in samples extracted in a water bath with boiling solvent during 30 and 60 minutes $(2.33$ and $1.95 \%$, respectively). Since extraction in a water bath is efficient and reduces a lot of time and solvent consumption, this method could present potential replacement for Pharmacopoeia-recommended Soxhlet extraction procedure.
\end{abstract}

Key words: Silybum marianum; silymarin; extraction method; UV spectroscopy; HPLC

http://dx.doi.org/10.5937/leksir1838005D

\section{INTRODUCTION}

Milk thistle, Silybum marianum (L.) Gaertn. (syn. Carduus marianum L.) Asteraceae, is a highly appreciated medicinal herb. There are certain data which can confirm the use of this plant since early times, for different diseases and disruptions of liver and gallbladder (hepatitis, cirrhosis and jaundice) and for the protection of the liver from the influence of alcohol and other harmful matters (Qavami et al., 2013; Blumenthal, 2003). Ancient Greek and Roman doctors were among the first to use milk thistle. The antique botanist Theophrastus named it "Pternix", Discorides named it "Sillybon", Pliny the Elder "Sillybum" and he recommended the juice of this plant mixed with honey for bile related complaints (Qavami et al., 2013).

Silibi mariani fructus is used as the medicinal part of the plant, and is included in the European Pharmacopeia and some national pharmacopeias (EMA, 2018a). All important monographs about medicinal plants and phytopreparations contain information about positive influence of milk thistle fruit preparations. According to EMA (2018b), the fruit of milk thistle is used as traditional herbal medicinal product for the symptomatic relief of digestive disorders, sensation of fullness and indigestion, as well as to support the liver function after serious conditions have been excluded by a doctor. According to ESCOP Monographs (2009) and WHO Monographs (2004) the fruit of milk thistle shows the stabilizing effect to the cell membranes of hepatocytes, anti-cholestatic, anti-inflammatory, antifibrotic, anti-carcinogenic effects, radical scavenging properties, effects on the regeneration of the liver and cellular metabolism. In in vivo experiments positive effects in acute and chronic intoxication of the liver have been described, anti-inflammatory effects, effects on the cardiovascular system, antinephrotoxic effects, anti-carcinogenic effects, as well as other effects such as anti-ulcerogenic and laxative effects. The monograph of German Commission E (Blumenthal et al., 1998) states that the crude drug is used with dyspeptic disorders, while phyto-preparations are used in toxic liver damage and in supportive treatment of chronic inflammatory liver conditions and liver cirrhosis.

Milk thistle fruit contain the following substances: flavonolignans (1.3-3\%) silybinin and isosilybinin (A and B), silycristin and silydianin that are collectivelly refered to as silymarin (Figure 1); flavonoids: flavones - apigenin, chrysoeriol, eriodictyol; flavonols - taxifolin, quercetin, dihydrokaempferol, kaempferol; fatty oil (20-30\%): linoleic (35-55\%) and oleic (24$30 \%$ ) acids, palmitic (8-12\%), linolenic (3-7\%), behenic (3-9\%) and other fatty acids; phytosterols (0.2-0.6\%): / beta-sitosterol; and other constituents (EMA, 2018a).

Herbal preparations for the oral use are generally made as water-ethanol extracts or with other organic solvents, where residues must be eliminated by special technological procedures. The efficacy of the extraction of medicinal plants in order to obtain the extracts with the maximal content of active principles highly depends on the method of extraction and extragens. There are conventional extractions such as maceration, digestion and percolation, the extraction at increased temperature on a water bath with the air cooler, and Soxhlet extraction or hot continuous extraction. Recently, the application in the preparation of different herbal extracts have found even more complex methods of extraction such as microwave as- 


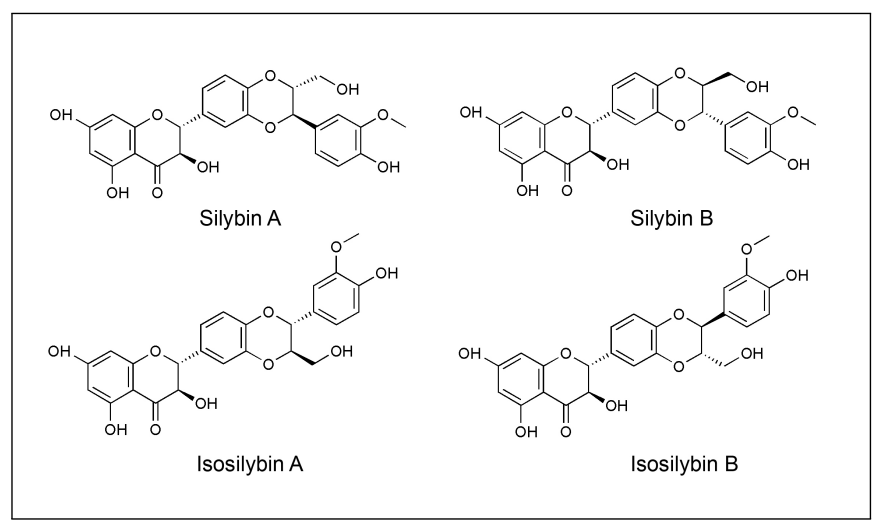

Fig. 1. Structures of the main flavonolignans from milk thistle fruit

sisted extraction (MAE), ultrasound-assisted extraction (UAE), supercritical fluid extraction (SFE), accelerated solvent extraction (ASE) and many others (Gupta et al., 2012; Azwanida, 2015). The knowledge of solubility of active chemical compound is of high importance for the selection of suitable extragens of certain polarity.

According to the literature data, the mode of extraction has a major influence on the silymarin content (Chambers et al., 2017). Ph. Eur. 7.0. (2010) recommends Soxhlet extraction with methanol for 5 hours. Instead of this time-consuming process and in order to reduce solvent requirements, other techniques such as maceration, ultrasound-assisted extraction, or pressurized liquid extraction have been proposed (Wianowska and Winiewski, 2015; Saleh et al., 2015). Water, and various organic solvents such as methanol, ethanol, acetone and ethyl acetate were tested for their ability to recover silymarin from milk thistle (Wallace et al., 2005; Poppe and Petersen, 2016). However, water and ethanol received special attention being non-toxic and eco-friendly solvents which is important for formulation of bioactive compounds. Experimental solubility data of silymarin in various solvents showed that the mole fraction solubility of silymarin in ethanol was much higher than in water (Shakeel et al., 2014). Bilia et al. (2000) reported that $60 \%$ aqueous ethanol was more effective solvent for extracting flavonolignans than $40 \%$ ethanol. Therefore, we used $60 \%$ ethanol as extraction solvent system in relation to this eco-friendly and sustainable biotechnological platform.

Different analytical methods have been used to determine silymarin content in Silybum marianum extracts, the most common being UV spectrophotometry (Meghreji et al., 2010) and HPLC (Mudge et al., 2015) methods. Spectrophotometric method is routine, simple and sensitive, but HPLC technique is more reliable and specific. The aim of the present work was to give more comprehensive research about which extraction conditions were the best for the efficient extraction of silymarin from milk thistle fruit. In this study, we investigated the effects of different extraction methods (maceration, percolation, water bath extraction, and ultrasonic-assisted extraction) on the content of silymarin in milk thistle fruit. UV/Vis and HPLC techniques were used for the determination of silymarin in obtained extracts.

\section{MATERIALS AND METHODS}

\subsection{Plant material}

Fruits from cultivated milk thistle were collected from the experimental field at Institute for Medicinal Plants Research "Dr Josif Pančić" in Pančevo, Serbia. The identification of plant material was carried out in the Laboratory for Pharmaceutical control of the Institute.

\subsection{Chemicals}

Silybin standard, and orthophosphoric acid were purchased from Sigma-Aldrich (Chemie $\mathrm{GmbH}$, Munich, Germany). Ethanol and methanol were of analytical grade, acetonitrile (Merck, Germany) was of HPLC grade, and ultra-pure water was prepared using a Milli-Q purification system (Millipore, France). Milk thistle dry extract was purchased from European Directorate for the Quality of Medicines and HealthCare (Strasbourg, France).

\subsection{Extraction procedures}

\subsubsection{Percolation}

Single percolation was carried out according to the Ph. Jug. IV (1984). Thirty g of grounded seeds of milk thistle were covered with $30 \mathrm{~mL}$ of solvent (1:1), and left for 2 hours to swell up. The saturated drug was put into the percolator and left for 24 $\mathrm{h}$ to macerate. The day after, the extract was easily separated and filtered.

\subsubsection{Maceration}

Maceration was carried out according to the Ph. Jug. IV (1984). Twenty g of the grounded seeds of milk thistle were covered with $100 \mathrm{~mL}$ of solvent (1:5), and in the following five days shaken few times per day. After the fifth day, the extract was filtered.

\subsubsection{Ultrasound-assisted extraction}

Five $g$ of the grounded seeds were extracted with $25 \mathrm{~mL}$ of solvent (1:5) in an ultrasonic bath (bath power $35 \mathrm{~W}$, continuous mode at frequency of $40 \mathrm{kHz}$, Maget USB 4, Bela Palanka, Serbia) at temperature of $40^{\circ} \mathrm{C}$ for 15 and $30 \mathrm{~min}$.

\subsubsection{Extraction on a water bath}

Five $g$ of the grounded seeds was mixed with $50 \mathrm{~mL}$ (1:10) of the solvent in $100 \mathrm{~mL}$ round-bottom flask and held in a water bath with the air cooler for 30 and 60 minutes at the boiling point of solvent.

\subsection{UV analysis}

A Hewlett Packard 400N spectrophotometer was used for estimation of silymarin in different samples. Reference standard of silybin was dissolved in methanol to obtain final concentration of $8 \mu \mathrm{g} / \mathrm{mL}$. Absorption of silybin and test solutions was detected at $288 \mathrm{~nm}$, and the content of silymarin in \% was expressed as silybin according to equation:

$$
\text { Silymarin }(\%)=\frac{A_{\text {sample }} \times m_{0}}{A_{0} \times m_{\text {sample }}} \times 100
$$

where $A_{\text {sample }}$ is absorption value of sample, $A_{0}$ is absorption value of silybin, $\mathrm{m}_{0}$ is mass of silybin, and $\mathrm{m}_{\text {sample }}$ is mass of the sample.

\subsection{HPLC analysis}

Total silymarin content was assessed according to the procedure described in Ph. Eur. 7.0. (2010), with slight modifications. Analyses were carried out on Agilent 1200 RR HPLC instrument (Agilent, Waldbronn, Germany), equipped with DAD detector, using reverse phase Zorbax SB-C18 (Agilent) analytical column $(150 \mathrm{~mm} \times 4.6 \mathrm{~mm}$ i.d., $5 \mu \mathrm{m}$ particle size $)$, and the column temperature was maintained at $30{ }^{\circ} \mathrm{C}$. The mobile phase was composed of (A) $1 \% \mathrm{v} / \mathrm{v}$ solution of orthophosphoric acid in water, and (B) acetonitrile. Gradient elution was applied according to the following scheme: $0-5$ $\min , 80-75 \%$ A; 5-30 $\mathrm{min}, 75 \%$ A; 30-35 $\mathrm{min}, 75-65 \%$ A; 35-40 min, $65-0 \% \mathrm{~A} ; 40-45 \mathrm{~min}, 0 \% \mathrm{~A}$. The flow rate of $0.8 \mathrm{~mL} / \mathrm{min}$, and injection volume was $10 \mu \mathrm{L}$. Detection wavelength was set at $288 \mathrm{~nm}$. A reference solution was prepared by exactly weighing $1 \mathrm{mg}$ of milk thistle dry extract dissolving in $1 \mathrm{~mL}$ 


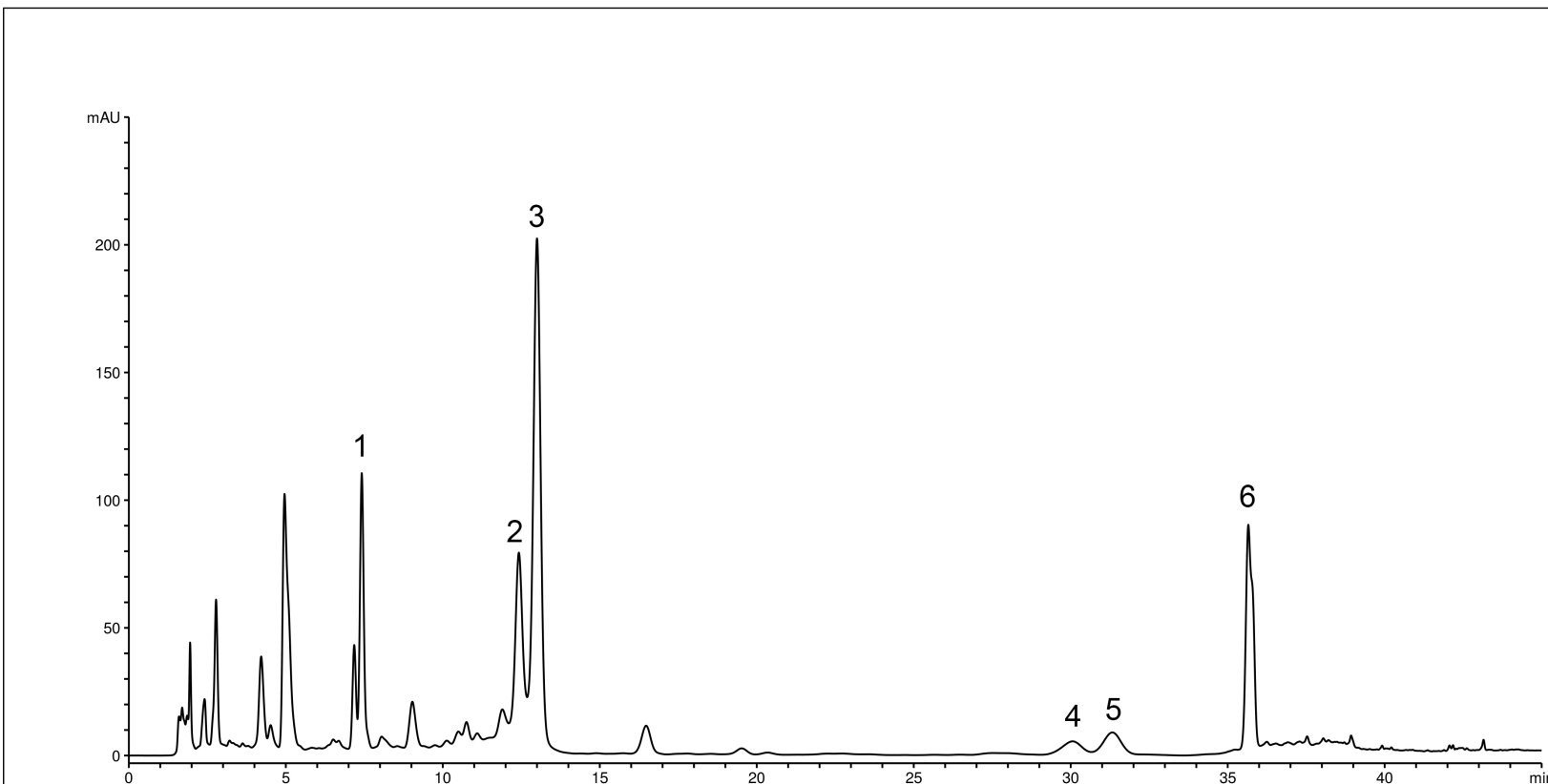

Fig. 2. HPLC chromatogram $(\lambda=288 \mathrm{~nm})$ of $60 \%$ ethanol extract of milk thistle fruit. Peaks: 1-taxifolin; 2-silychristin; 3-silydianin; 4-silybin A 5-silybin B; 6-isosilybin

of methanol. The amount of total silymarin in percentage $(\%)$, expressed as silybin, was calculated from the expression:

$$
\text { Silymarin }(\%)=\frac{A_{\text {sample }} \times m_{0}}{A_{0} \times m_{\text {sample }}} \times 100
$$

where $\mathrm{A}_{\text {sample }}$ is area of flavonolignans peaks in sample, $\mathrm{A}_{0}$ is area of silybin peak in reference solution, $\mathrm{m}_{0}$ is mass of silybin in reference solution, and $\mathrm{m}_{\text {sample }}$ is mass of the sample.

\section{RESULTS AND DISCUSSION}

Silymarin content in various samples obtained by different extraction techniques was analyzed simultaneously by UV and HPLC methods, and results are presented in Table 1. HPLC chromatogram of extract obtained using water bath extraction is shown in Figure 2. The similarity between the employed analytical methods regarding silymarin content can be observed. According to the both HPLC and UV method, the highest amount was determined in samples extracted with boiling solvent during 30 and 60 minutes (2.33 and $1.95 \%$, respectively, by HPLC, and 3.60 and $3.13 \%$, respectively, by UV). Extract obtained using maceration contained moderate amount of silymarin $(0.54 \%)$, followed by samples obtained by UAE (ultrasonic-assisted extraction) during 15 and $30 \mathrm{~min}$ ( 0.25 and $0.46 \%$, respectively). The lowest content was determined in samples extracted by percolation technique $(0.15 \%)$. Obtained results clearly showed that extraction procedure had a major influence on the silymarin content, and temperature and extraction time were the main parameters which affected the extraction efficiency of flavonolignans. According to the literature data, extraction with hot water at $100{ }^{\circ} \mathrm{C}$ yielded high level of silymarin, whereas organic solvents such as ethanol or methanol gave better results at their sub-boiling temperatures at $60{ }^{\circ} \mathrm{C}$ (Wallace et al., 2005). In our study, highest concentration of silymarin was measured after extraction in water bath at $85{ }^{\circ} \mathrm{C}$ which is a boiling temperature of $60 \%$ ethanol. Extraction in a longer time (60 minutes) slightly decreased the efficiency probably due to thermal degradation of the analytes. In the case of another conventional method, maceration, extracted amount of silymarin was slightly higher than the val-
Table 1. The content of silymarin (\%) in various extracts determined by UV and HPLC methods

\begin{tabular}{lcr}
\hline Extraction technique & UV & HPLC \\
\hline Percolation & 0.95 & 0.15 \\
Maceration & 2.36 & 0.54 \\
UAE $15 \mathrm{~min}$ & 0.58 & 0.25 \\
UAE 30 min & 0.97 & 0.46 \\
Water bath $30 \mathrm{~min}$ & 3.60 & 2.03 \\
Water bath $60 \mathrm{~min}$ & 3.13 & 1.94 \\
\hline
\end{tabular}

ues obtained by UAE. When extraction was performed with UAE, considerably higher yield was achieved during longer extraction time (30 $\mathrm{min})$. Saleh et al. (2015) also reported that increasing ultrasonic-assisted extraction time from 15 to 60 min increased the yield of the silymarin.

Our data regarding silymarin concentrations obtained by extraction in a water bath are in accordance with the requirements of Ph. Eur. 7.0. (2010) that prescribes a minimum of 1.5\% of silymarin in milk thistle fruit. Quaglia et al. (1999) reported slightly higher values of silymarin than in our study, which could be the consequence of different extraction conditions used in the experiments.

\section{CONCLUSION}

In the present study, the efficiency of different extraction techniques in silymarin recovery from milk thistle fruit was tested. The obtained results showed that the highest concentration of silymarin was measured in sample extracted in a water bath at $85{ }^{\circ} \mathrm{C}$ for $30 \mathrm{~min}$ with $60 \%$ ethanol. This method is suitable for silymarin extraction due to its efficiency and reduction of time and solvent consumption, and could present potential replacement for Pharmacopoeia-recommended Soxhlet extraction procedure. 


\section{ACKNOWLEDGMENTS}

Acknowledgment.This work was supported by the Ministry of Education, Science and Technological Development of the Republic of Serbia, project No. 46013 and No 45017.

\section{REFERENCES}

Azwanida, N. (2015). A Review on the Extraction Methods Use in Medicinal Plants, Principle, Strength and Limitation, Medicinal and Aromatic Plants 4(3): 196-202.

Bilia, A. R., Salvini, D., Mazzi, G. and Vincieri, F. F. (2000) Characterization of calendula flower, milk-thistle fruit, and passion flower tinctures by HPLC-DAD and HPLC-MS, Chromatographia 53(3): 210-215.

Blumenthal, M., Busse, W. R., Council, A. B., Communications, I. M. and E, G. B. C. (1998). The complete German Commission E monographs : therapeutic guide to herbal medicines, Austin, Tex. : American Botanical Council ; Boston, Ma. : Integrative Medicine Communications.

Blumenthal, M. (ed.) (2003). The ABC Clinical Guide to Herbs, 1st edition edition edn, American Botanical Council, Austin, Tex.

Chambers, C. S., Holečková, V., Petrásková, L., Biedermann, D., Valentová, K., Buchta, M. and Křen, V. (2017). The silymarin composition... and why does it matter???, Food Research International 100: 339-353.

EMA (2018a). Assessment report on Silybum marianum (1.) gaertn., fructus, European Medicines Agency.

EMA (2018b). European union herbal monograph on Silybum marianum (1.) gaertn., fructus, European Medicines Agency.

ESCOP Monographs (2009). European Scientific Cooperative on Phytotherapy, supplement edition edn, Thieme, Stuttgart, Exeter.

Gupta, A., Naraniwal, M. and Kothari, V. (2012). Modern Extraction Methods for Preparation of Bioactive Plant Extracts, International Journal of Applied and Natural Sciences 1(1): 8-26.

Meghreji, M., Patel, N., Dave, B., Badmanaban, J. and A.J., P. (2010). Validated method for Silymarin by Spectrophotometry in Bulk drug and Pharmaceutical formulations, Journal of Chemical and Pharmaceutical Research 2(1).

Mudge, E., Paley, L., Schieber, A. and Brown, P. N. (2015). Optimization and single-laboratory validation of a method for the determination of flavonolignans in milk thistle seeds by high-performance liquid chromatography with ultraviolet detection, Analytical and Bioanalytical Chemistry 407(25): 7657-7666

Ph. Eur. 7.0. (2010). European Pharmacopoeia 7.0., Council of Europe, Strasbourg.

Ph. Jug. IV (1984). Yugoslav Pharmacopoeia IV, Savezni zavod za zdravstvenu zaštitu, Beograd. Google-Books-ID: w1IWYAAACAAJ.

Poppe, L. and Petersen, M. (2016). Variation in the flavonolignan composition of fruits from different Silybum marianum chemotypes and suspension cultures derived therefrom, Phytochemistry 131: 68-75.

Qavami, N., Naghdi Badi, H., Labbafi, M. and Mehrafarin, A. (2013). A review on pharmacological, cultivation and biotechnology aspects of milk thistle (silybum marianum (1.) gaertn.), Journal of Medicinal Plants 12(47): 19-37.
Quaglia, M., Bossù, E., Donati, E., Mazzanti, G. and Brandt, A. (1999). Determination of silymarine in the extract from the dried silybum marianum fruits by high performance liquid chromatography and capillary electrophoresis, Journal of Pharmaceutical and Biomedical Analysis 19(3-4): 435-442.

Saleh, I. A., Vinatoru, M., Mason, T., Abdel-Azim, N., Aboutabl, E. and Hammouda, F. (2015). Ultrasonic-assisted extraction and conventional extraction of Silymarin from Silybum marianum seeds; a comparison, Research Journal of Pharmaceutical Biological and Chemical Sciences 6(2): 709-717.

Shakeel, F., Anwer, M., Shazly, G. and Jamil, S. (2014). Measurement and correlation of solubility of bioactive compound silymarin in five different green solvents at $298.15 \mathrm{k}$ to 333.15k, Journal of Molecular Liquids 195: 255-258.

Wallace, S., Carrier, D. and Clausen, E. (2005). Batch solvent extraction of flavanolignans from milk thistle (Silybum marianum L. Gaertner), Phytochemical Analysis 16(1): 7-16.

WHO Monographs (2004). WHO Monographs on selected medicinal plants, Vol. 2, World Health Organization, Geneva, chapter Fructus Silybi Mariane.

Wianowska, D. and Winiewski, M. (2015). Simplified Procedure of Silymarin Extraction from Silybum marianum L. Gaertner, Journal of Chromatographic Science 53(2): 366-372. 